\title{
Adaptive Stokes Space Based Polarization Demultiplexing for Flexible UDWDM Metro-Access Networks
}

\author{
Somayeh Ziaie ${ }^{(1)}$, Nelson J. Muga ${ }^{(1)}$, Ricardo M. Ferreira ${ }^{(1)}$, Fernando P. Guiomar ${ }^{(2)}$, Ali Shahpari ${ }^{(1)}$, \\ António L. Teixeira ${ }^{(1)}$ and Armando N. Pinto ${ }^{(1)}$ \\ (1) DETI, Universidade de Aveiro, Instituto de Telecomunicações, 3810-193, Aveiro, Portugal. \\ (2) Dipartimento di Elettronica e Telecomunicazioni, Politecnico di Torino, Corso Duca degli Abruzzi, 24, 10129 Torino, Italy.
}

\begin{abstract}
We experimentally demonstrate a flexible coherent UDWDM system with support to optical-wireless links and adaptive DP-QPSK/DP-16QAM modulation, enabled by Stokes-based polarization-demultiplexing. The system is shown to be resilient to dynamic power ranges of $>12 \mathrm{~dB}$. OCIS codes: (060.1660) Coherent communications, (060.2605) Free-space optical communication, (060.4256) Networks.
\end{abstract}

\section{Introduction}

Due to the plurality of end user types and the dynamic nature of mobile networks, metropolitan and access networks are evolving to provide improved availability, data rates and services. Sophisticated and high-performance transceivers are required to support a very-high capacity, while still guaranteeing other key features of optical metroaccess networks, such as load balancing, low latency and resiliency [1]. However, in order to meet commercial viability, the quest for flexible and spectrally efficient optical metro-access networks must follow the path of simple and robust solutions using advanced optical components and systems as well as optimization of the network concept, ensuring a dramatic reduction of the cost per bit.

Using bidirectional mesh configurations as the one shown in Fig. 1, all-optical merged networks with different capacities, bandwidths and adaptive distances can be connected, enabling fast and efficient provisioning of high data rate optical paths. In this context, support to flexible grids and data rates, as well resiliency to high dynamic power range (DPR) are increasingly important issues for the design of this kind of networks. Meanwhile, free space optics (FSO) links have attracted great attention within the research community as a temporary, fast and cost-effective solution for the network resiliency without limitation in bandwidth and type of the network configuration [2]. In addition, in optical metro networks based on mesh topology, any signal with different optical powers and modulation formats can be added to the system according to the load of the network. Therefore, the performance of high spectral efficiency (SE) systems in a flexible network should be characterized.

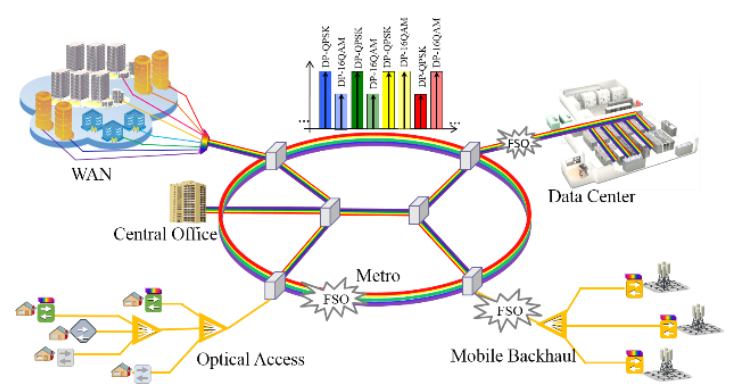

Fig. 1. Exemplary topology of a flexible optical metro-access network.

It has been shown that coherent ultra-dense wavelength division multiplexing (UDWDM) together with dual polarization (DP) pulse shaped multi-level modulation formats and digital signal processing (DSP) enable high SE, excellent sensitivity and selectivity in optical metro-access networks [2-4]. Current polarization demultiplexing (PolDemux) techniques based on the constant modulus algorithm (CMA) can effectively be employed in DSP, but at the price of data format dependent operation and being liable to suffer from singularity issues when operating in blind mode [4]. Alternatively, an adaptive Stokes space technique has been recently reported as an advantageous PolDemux method for optical networks characterized by low accumulated chromatic and polarization mode dispersion, providing modulation format transparency, as well as higher immunity to phase noise, faster convergence and avoidance of singularity issues $[5,6]$.

In this paper, we evaluate the performance of a flexible optical link based on Nyquist pulse shaping UDWDM (6.25 GHz ITU-T grid) implemented by DP modulation formats and DSP based on the adaptive Stokes PolDemux algorithm. The hybrid optical signals based on DP-QPSK and DP-16QAM modulation formats are transmitted over up to $80 \mathrm{~km}$ of standard single-mode fiber (SSMF) plus $54 \mathrm{~m}$ outdoor FSO link, as well as field-deployed fiber. The system performance in terms of sensitivity and resilience to DPR is assessed by counting the bit error rate (BER).

\section{Experimental Setup}

In Fig. 2 (a), we show the experimental setup proposed in this paper to characterize a flexible hybrid bit rate and distribution system based on Nyquist shaping UDWDM and the adaptive Stokes PolDemux method. At the transmitter, the light from a $100 \mathrm{kHz}$ linewidth external cavity laser (ECL) was injected in a Mach-Zehnder modulator 


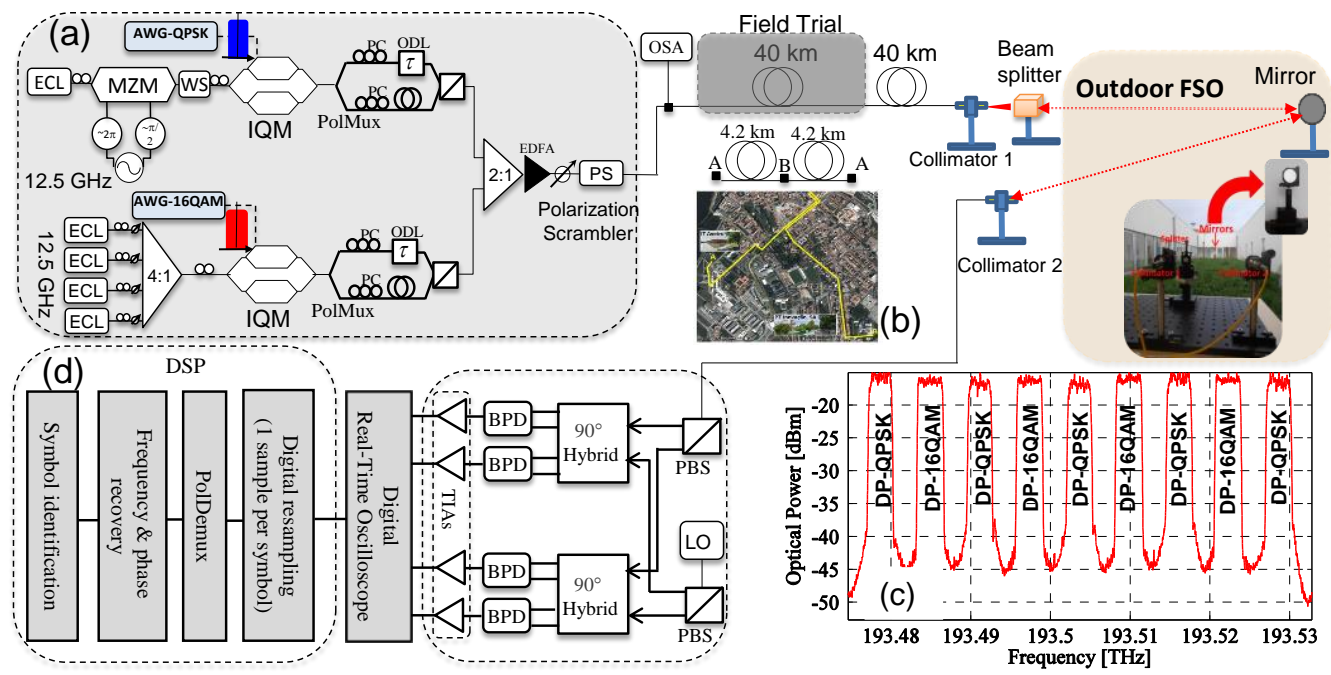

Fig. 2. (a) Experimental setup for flexible transmission up to 80-km SSMF and FSO; (b) Field-trial connection; (c) Spectrum of 3.125 Gbaud DP-QPSK and DP-16QAM UDWDM channels with $6.25 \mathrm{GHz}$ channel spacing; (d) DSP subsystems.

(MZM) driven by $12.5 \mathrm{GHz}$ RF signals to generate multi-tones. Using a wavelength selective switch (WS), 5 flat channels were filtered and selected. These 5 tones were fed into an IQ modulator (IQM), driven by a $12 \mathrm{GSa} / \mathrm{s}$ arbitrary waveform generator (AWG), producing a 3.125 Gbaud QPSK or 16QAM signal $\left(2^{15}\right.$ pseudo random binary sequence (PRBS)) and digitally filtered by a raised-cosine filter with 0.1 roll-off factor. The signal was separated into two polarizations (69 symbols delayed for de-correlation purposes) using an optical delay line (ODL), and then multiplexed in polarization again by a polarization beam combiner, creating dual polarization 12.5 and $25 \mathrm{~Gb} / \mathrm{s} \mathrm{DP}$ QPSK and DP-16QAM, respectively. Combining 4 ECL lasers with $12.5 \mathrm{GHz}$ frequency spacing and feeding it to the second IQM driven by a $65 \mathrm{GSa} / \mathrm{s}$ AWG, $4 \times 3.125$ Gbaud QPSK or 16QAM signals were generated. In the PolMux part, these signals were polarization multiplexed and then interleaved with the previous 5 channels by means of a coupler. The optical spectrum of these 9 channels is shown in Fig. 2 (c). The launch power at the fiber input for 9 channels was set by an erbium-doped fiber amplifier (EDFA) and a variable optical attenuator (VOA) prior to the polarization scrambler and the transmission link. The transmission part was based on $80 \mathrm{~km}$ fiber, field trial plus 40 $\mathrm{km}$ fiber and $80 \mathrm{~km}$ plus FSO. We used installed fiber in Aveiro city, Portugal (satellite view in Fig. 2 (b)), plus 40 $\mathrm{km}$ of SSMF in the lab. The FSO link with total loss of $8 \mathrm{~dB}$ was based on $54 \mathrm{~m}$ outdoor optical wireless link that included two collimators, polarization independence beam splitter and convex mirror. Using a beam splitter we were able to divide the FSO link into two links. Note that we only analyze the straight direction as shown in Fig. 2 (a). At the receiver side, UDWDM signals were detected using an integrated phase- and polarization-diverse coherent receiver followed by a $50 \mathrm{GSa} / \mathrm{s}$ real-time scope. An ECL with $13 \mathrm{dBm}$ optical power and $100 \mathrm{kHz}$ linewidth was used as a local oscillator.

The DSP chain was implemented offline [6] using MATLAB, and comprised digital resampling, PolDemux, and frequency and carrier phase recovery subsystems, as depicted in Fig. 2 (d). The electrical output signal was resampled to 1 sample per symbol. In order to accomplish a robust, flexible and format-transparent separation of the mixed signals transmitted in the two orthogonal polarizations, we used the adaptive Stokes space based PolDemux algorithm. By representing data in the Stokes space, the algorithm finds a best fitting plane, whose normal is then employed into the computation of the inverse polarization rotation matrix of the channel. Sample by sample, and agnostically to the modulation format, the orientation of the normal $\hat{n}(k)$ is therefore updated following the rule $\hat{n}(k+1)=$ $(\hat{n}(k)+\vec{\Gamma}(k+1)) B$, with B representing a normalization factor, and $\vec{\Gamma}(k+1)=\eta A[\hat{s}(k+1) \times \hat{n}(k)] \times \hat{n}(k)$, where $\eta$ is the step-size parameter and $\hat{s}(k+1)$ is the Stokes vector of the optical field at sample $k+1$.

\section{Experimental Results and Discussion}

In order to verify and assess the performance of the proposed adaptive Stokes algorithm, Fig. 3 (a) shows the measured BER as a function of the receiver input power per multi-channel for back-to-back (BTB), transmission scenarios over $80 \mathrm{~km}$ fiber, FSO links and field-trial. The launched optical power per channel in the input of fiber was set to $-5 \mathrm{dBm}$. Results show an acceptable -45 and $-32 \mathrm{dBm}$ sensitivity considering a FEC-compatible BER $=3.8 \times 10^{-3}$ for DP-QPSK 

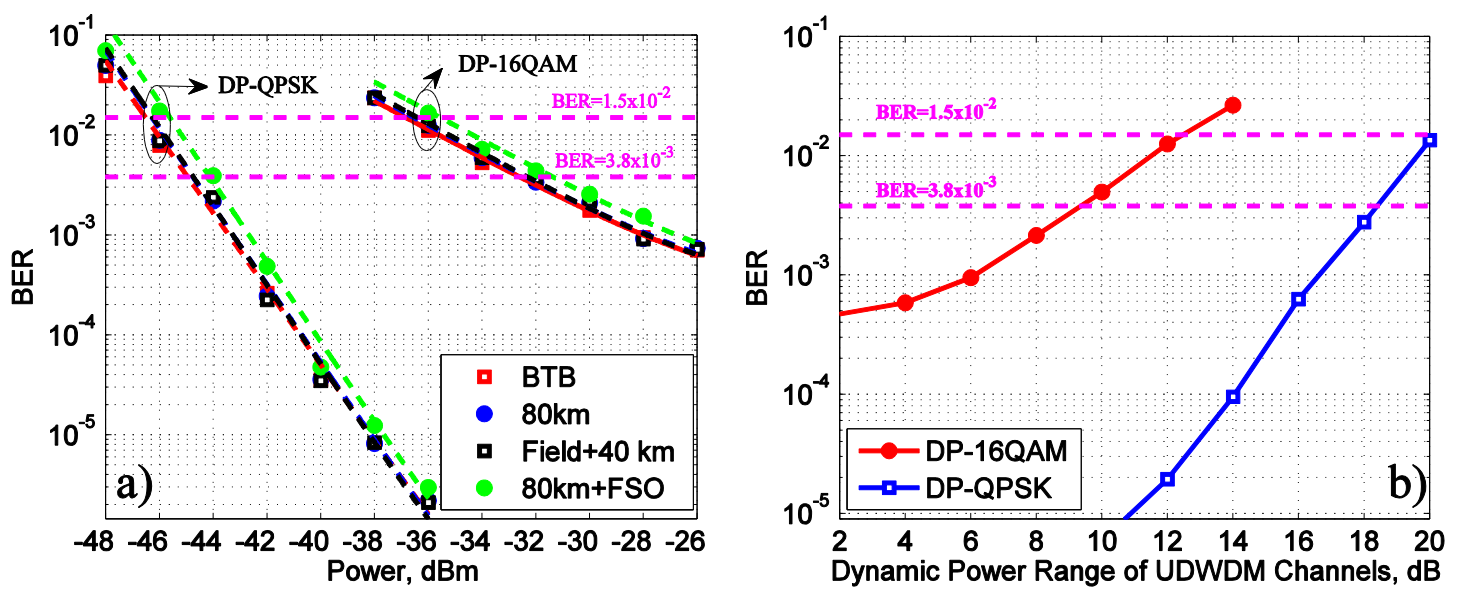

Fig. 3. (a) BER performance versus received optical power for $12.5 \mathrm{~Gb} / \mathrm{s}$ DP-QPSK and $25 \mathrm{~Gb} / \mathrm{s}$ DP-16QAM in the BTB, $80 \mathrm{~km}, 80 \mathrm{~km}$ plus FSO and field trial transmissions; (b) Measured BER for the central channel of UDWDM channels vs. DPR in the input of $80 \mathrm{~km}$ fiber.
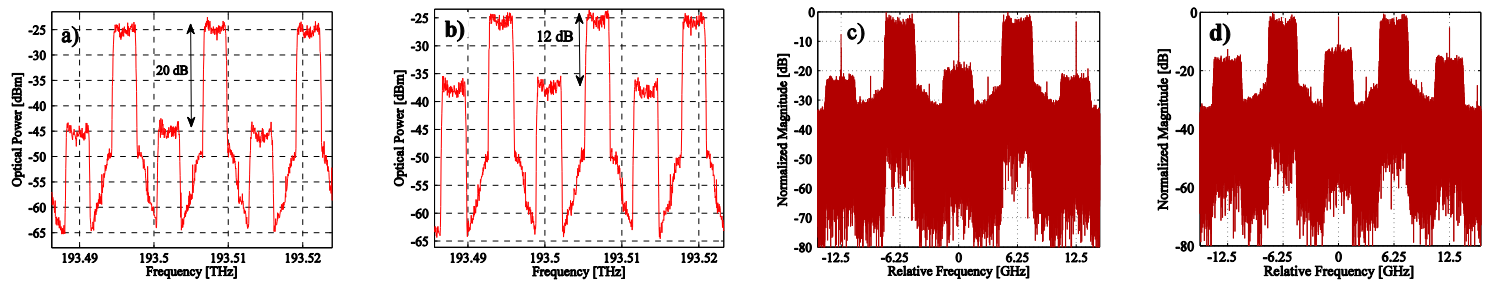

Fig. 4. Optical spectra in the input of fiber for (a) DP-QPSK channels (b) DP-16QAM channels; the electrical spectra after the DSP for (c) $20 \mathrm{~dB}$ DPR of DP-OPSK channels and (b) $12 \mathrm{~dB}$ DPR of DP-16OAM channels.

and DP-16QAM channels, respectively. For the hybrid optical wireless scenario, due to fluctuation of optical power, we observed $1 \mathrm{~dB}$ penalty in receiver sensitivity. Using soft-decision FEC $\left(1.5 \times 10^{-2}\right)$, the receiver sensitivities of -46 and $-36 \mathrm{dBm}$ are achieved for $10 \mathrm{~Gb} / \mathrm{s}$ DP-QPSK and $20 \mathrm{~Gb} / \mathrm{s}$ DP-16QAM, respectively.

Fig. 3 (b) depicts the DPR of the system, which characterizes the maximum power unbalance between adjacent channels. For this purpose, we fixed the transmitted optical power of DP-QPSK/ DP-16QAM channels and changed the launched power of the DP-16QAM/DP-QPSK signals. The difference between powers gives the DPR. Considering soft-decision FEC, the BER measurements present 20 and $12 \mathrm{~dB}$ DPR between channels for DP-QPSK and DP16QAM channels, respectively. Fig. 4 (a)-(b) present the optical spectra in the input of fiber and the electrical spectra after the DSP for DP-QPSK channels with $20 \mathrm{~dB}$ DPR and DP-16QAM channels with $12 \mathrm{~dB}$ DPR.

\section{Conclusions}

We have experimentally demonstrated a dynamic bandwidth and power range optical metro and access network, considering back-to-back and transmission scenarios over $80 \mathrm{~km}$ fiber, FSO links and field-trial. Hybrid DP-QPSK and DP-16QAM modulation is enabled by format-transparent Stokes space PolDemux, simultaneously providing high sensitivity and flexibility with reasonable dynamic power range at $6.25 \mathrm{GHz}$ channel spacing. Performance in field trial was also evaluated in terms of sensitivity confirmed by the penalty-free comparison with the laboratory results. Allying its fast convergence speed and format-transparency, the adaptive Stokes space PolDemux is a promising technique to be used in reconfigurable DSP for flexible optical metro-access networks, as well as optical wireless scenarios.

This work was partially supported by FCT/MEC through national funds and when applicable co-funded by FEDER-PT2020 partnership agreement under the project UID/EEA/50008/2013 (action SoftTransceiver and Optical-5G), the FCT grants SFRH/BPD/77286/2011 and FRH/BPD/110889/2015 and by the European Commission through project Flex-ON (653412).

\section{References}

[1] C. Rottondi et al., "Optical ring metro networks with flexible grid and distance-adaptive optical coherent transceivers," Bell Labs J., 18, p. 95 2013.

[2] A. Shahpari et al., "Coherent ultra-dense wavelength division multiplexing passive optical networks," Optical Fiber Techn., 26, p.100, 2015.

[3] D. Lavery et al, "Digital coherent receivers for long-reach optical access networks", J. Lightwave Technol., 31, p.609, 2013.

[4] S. Savory, "Digital coherent optical receivers: algorithms and subsystems," IEEE J. Sel. Topics Quantum Electron. 16, p. 1164, 2010.

[5] N. J. Muga et al.,"Adaptive 3-D Stokes space-based polarization demultiplexing algorithm," J. Lightwave Technol., 32, p. 3290 , 2014.

[6] S. Ziaie, et al., "Experimental assessment of the adaptive Stokes space-based polarization demultiplexing for optical metro and access networks," J. Lightwave Technol., 33, p. 4968, 2015. 